American Journal of Immunology 1 (2): 74-78, 2005

ISSN 1553-619X

(c) 2005 Science Publications

\title{
Comparative Evaluation of Aqueous and Freeze Dried Antigen for Sero-diagnosis of VL and PKDL Cases by DAT
}

\author{
${ }^{1}$ Sanjiva Bimal, ${ }^{1}$ Shubhankar K. Singh, ${ }^{1}$ P. K. Sinha, ${ }^{2}$ P. K. Das, ${ }^{2}$ H. Schallig \\ ${ }^{3} \mathrm{P}$. Das and ${ }^{1} \mathrm{~S}$. K. Bhattacharya \\ ${ }^{1}$ Division of Immunology, Rajendra Memorial Research Institute of Medical Sciences \\ Agamkuan, Patna, India, 800007 \\ ${ }^{2}$ N.H.-Swellengrebel-laboratory for Tropical Hygiene Meibergdreef 391105 AZ Amsterdam \\ The Netherlands \\ ${ }^{3}$ National Institute of Cholera and Enteric Diseases, P-33, CIT Road, Scheme XM \\ Beliagahta, Kolkata, India, 700010
}

\begin{abstract}
The efficacy of aqueous and freeze dried (FD) antigen was evaluated by direct agglutination test (DAT) for sero-diagnosis of visceral leishmaniasis. A total of 16 samples collected from 5 confirmed Kala-azar (KA) cases, 6 cases 2 each of filariasis, malaria, leprosy and 5 non-endemic healthy subjects were used in determination of cut off titre of both the antigens in DAT. A 1:800 for aqueous and 1:400 dilution for FD DAT antigen was found as cutoff values for differential diagnosis of KA cases from control subjects. Both antigens revealed $100 \%$ correlation in terms of sensitivity and specificity in diagnosis of KA cases and healthy subjects at these titres. Using both antigens and the cut off titres, sera from 26 individuals collected from different categories of leishmaniasis viz. Kala-azar pre-treatment 10, Kala-azar post-treatment 10, HIV-KA co-infection 1 and PKDL 5 were evaluated by DAT. Both the antigens showed equal sensitivity and specificity in diagnosis of different categories of leishmaniasis. The study concludes that though aqueous antigen showed equal sensitivity and specificity but $\mathrm{FD}$ antigen which is stable at $56^{\circ} \mathrm{C}$ do not require cold chain whereas aqueous antigen is unstable at room temperature and require proper cooling to retain its antigenic activity as studied in between day 1 and day 14, so the FD antigen should be used for routine diagnosis of KA cases particularly in field condition.
\end{abstract}

Key words: Kala-azar cases, aqueous and freeze dried antigen, field condition, DAT

\section{INTRODUCTION}

Although direct demonstration of Leishmania donovani parasite in the biopsy smear or culture imprints of spleen or other biopsy materials are the gold standards for diagnosis of Kala-azar, but these procedures are invasive and lack screening capabilities of large number of samples particularly during epidemics or in endemic situations. These conventional methods are being replaced by indirect methods such as serological and DNA based diagnostics ${ }^{[1]}$. Serological tests such as enzyme linked immunosorbent assays (ELISA), immunofluorescent antibody tests (IFATs), Western blot and the direct agglutination test (DAT) are currently in use to detect anti-Leishmania antibodies in samples of patients suspected of suffering from visceral leishmaniasis (VL) in rural areas. During the last decade, DAT has been considered as one of the simple, non-invasive and rapid technique with high sensitivity and specificity in rural areas ${ }^{[2-4]}$. The potential of DAT with respect to its application in remote areas has been improved by the development of heat stable freezedried version of $\mathrm{DAT}^{[5]}$.

The present study describes a comparative evaluation of the DAT using aqueous and FD antigen for the detection of anti-Leishmania antibodies in serum samples from Indian Kala-azar and Post Kala-azar dermal Leishmaniasis from Indian state of Bihar, along with other diseases and healthy controls.

\section{MATERIALS AND METHODS}

A laboratory adapted WHO reference strain of L. donovani (WHOM/IN/80/DD8) was used to prepare the aqueous DAT antigen ${ }^{[2]}$. Briefly, promastigotes were grown in monophasic media with $20 \%$ foetal calf serum (FCS). Ninety-six hours old promastigotes in stationary phase (approximately $50 \times 10^{6}$ promastigotes $\mathrm{mL}^{-1}$ ) were harvested and washed twice with Locke's solution (154mM NaCl, 2mM CaCl $2,2 \mathrm{mM} \mathrm{NaHCO}, 0.25 \%$ Glucose). Following trypsin treatment $(0.4 \%)$, the harvested promastigotes were washed twice with

Corresponding Author: Dr. S. Bimal, Division of Immunology, Rajendra Memorial Research Institute of Medical Sciences (ICMR), Agamkuan, Patna, India, Pin Code: 800007, Tel.: 0091-0612-2638680, Fax: 0091-0612-2644379 
Locke's solution. Promastigotes were fixed in $2 \%(\mathrm{w} / \mathrm{v})$ formaldehyde in Locke's solution for 18-20h. After washing in citrate saline $(0.5 \mathrm{M} \mathrm{NaCl}, 0.05 \mathrm{M}$ Sodium Citrate, $\mathrm{pH}$ 7.4), the promastigotes were stained with $0.02 \%$ Coomassie Brillant Blue. The washed parasites were suspended at a concentration of $7.5 \times 10^{7} \mathrm{~mL}^{-1}$ in citrate saline solution with $0.43 \%$ (w/v) formaldehyde before being preserved at $4^{\circ} \mathrm{C}$ until used. The freezedried antigen was procured from Biomedical Research Lab. Amsterdam, The Netherlands. That antigen remains stable at ambient temperature $\left(20^{0}-45^{\circ} \mathrm{C}\right)$ for at least 1 year was used in the study. Blood was collected on filter paper (already standardized at Rajendra Memorial Research Institute of Medical Sciences of Indian Council of Medical Research). Altogether 42 blood samples were tested of which 16 sera samples were tested for cut off titre determination which included 5 confirmed KA cases, 6 cases 2 each of filariasis, malaria, leprosy and 5 non-endemic healthy subjects.

A total of 26 subjects from following groups were later evaluated for comparative efficacy of aqueous and FD antigens which included

* Patients with clinically and parasitologically confirmed active VL $(\mathrm{n}=10)$

* Patients with HIV-VL co-infection ( $\mathrm{n}=1$ )

* Patients with Post Kala-azar Dermal Leishmaniasis $(n=5)$

* Patients treated with anti-leishmanial therapy for VL $(n=10)$. Samples were obtained 1-3 months after treatment

* Patients with parasitic diseases other then leishmaniasis $(n=6)$ comprising 2 cases of Malaria,Filariasis and Leprosy each.

* Non-endemic healthy control $(\mathrm{n}=5)$.

Sera were eluted from filter paper ${ }^{[6]}$. One round of blood on Whatman Filter paper No. 3 (equivalent to $5 \mu \mathrm{l}$ of blood) was immersed in $250 \mu$ l elution buffer $(0.9 \%$ $\mathrm{NaCl}, \mathrm{pH}$ 7.4). The eluted sera were equivalent to $1: 50$ dilution. The test was performed in V-shaped 96 well micro-titre plates (NUNC). Serum samples were serially two-fold diluted with saline- $0.2 \%(\mathrm{w} / \mathrm{v})$ gelatin solution, the initial dilution being 1:100. Negative control wells received $50 \mu \mathrm{l}$ saline with $0.2 \%$ gelatin solution. For screening with FD DAT antigen, one circle of blood on filter paper were added into the first column of the wells and sera eluted from blood for 8 hours at $4^{\circ} \mathrm{C}$. Fifty (50) $\mu \mathrm{l}$ of serum diluent fluid (Normal Saline) was added to all other wells of the plate. Fifty (50) $\mu \mathrm{l}$ of the eluted blood was transferred in the adjacent wells, mixed and again $(50 \mu \mathrm{l})$ transferred to the next well. The first dilution in the second well was 1:100. Serum dilution was repeated up to 1:102400 final dilutions. Fifty (50) $\mu \mathrm{l}$ of stained parasite suspension of aqueous or FD antigen were to all the wells after gentle shaking. The plate was rotated on a level surface for 30 seconds and incubated at room temperature for $18 \mathrm{~h}$. The test was read visually and titre was expressed as the highest dilution of serum, which showed definite agglutination of the parasite.

To detect the cut-off limit, sera from 5 confirmed VL cases and 5 healthy subjects from non-endemic areas were used. The proven VL cases were selected based on positive parasite in bone marrow/splenic aspirate and showing symptoms of splenomegaly and fever of minimum two weeks duration. All healthy subjects did not present any of these symptoms at the time of blood collection. The specificity of DAT was evaluated with sera samples from 6 patients with other infections, which comprised two each of leprosy, filariasis and malaria. Sensitivity was defined as proportion of the sick subjects, which were identified as positive as such by positive results.

$$
\begin{aligned}
& \text { True positive } \\
& \text { Sensitivity = --------------------------- }
\end{aligned}
$$

Specificity was defined as proportion of healthy subjects confirmed as such by the negative results.

$$
\begin{aligned}
& \text { True Negative } \\
& \text { Specificity }=\text {--orue Negative }+ \text { False Positive }
\end{aligned}
$$

Determination of antigenic activity at varied temperature: Prior to evaluating the specific role of these antigens in the diagnosis of different categories of VL patients, we studied the comparative effect of heat stability on anti leishmanial antibody titre. The FD and aqueous antigens were kept at $4^{\circ} \mathrm{C}$ and at room temperature and tested on VL positive and negative sera day-zero and day-14 in order to test performance of these antigens at altered temperatures during different periods of times.

Once the operational cut-off value was determined with test sensitivity and specificity analysis performed and heat stability testing conducted, 26 serum samples from different groups were chosen for comparative evaluation of aqueous and freeze dried version of DAT for diagnosis of Kala-azar and PKDL. These sera samples comprised of patients parasitologically proved VL $(\mathrm{n}=10), 10$ patients who had been treated for VL (7 inj. fungizone $\mathrm{N}=1,15 \mathrm{inj}$. Fungizone $\mathrm{N}=3,30 \mathrm{inj}$ of $\mathrm{SAG} N=5$ and $60 \mathrm{inj}$. of SAG N=1) in the past, 5 PKDL cases with duration of illness ranging from 5 months to 3years.

\section{RESULTS AND DISCUSSION}

The cut off titre for any test varies from lab to lab even with the same antigen or different antigen. To determine the cutoff titre of DAT for both antigens, sera from clinically postive cases of Kala-azar and control subjects from Kala-azar non-endemic areas were 
Table 1: Comparison of DAT titre* obtained with aqueous and freeze dried antigen in clinically positive cases of Kala-azar and control

\begin{tabular}{|c|c|c|c|c|c|c|c|c|c|c|c|}
\hline \multirow[t]{2}{*}{ Groups } & \multirow[t]{2}{*}{$\begin{array}{l}\text { Ag. } \\
\text { Used }\end{array}$} & \multicolumn{10}{|c|}{$\begin{array}{l}\text { DILUTIONS } \\
(1 / \text { titre })\end{array}$} \\
\hline & & $<100$ & 100 & 200 & 400 & 800 & 1600 & 3200 & 6400 & 12800 & $\geq 25600$ \\
\hline \multirow{2}{*}{$\begin{array}{l}\text { Kala-azar } \\
(\mathrm{n}=5)\end{array}$} & Aq. & - & 5 & 5 & 5 & 5 & 4 & 2 & 2 & 1 & - \\
\hline & FD & - & 5 & 5 & 5 & 5 & 3 & 2 & 2 & 0 & - \\
\hline \multirow{2}{*}{$\begin{array}{l}\text { Filaria } \\
(\mathrm{n}=2)\end{array}$} & Aq. & - & 2 & 1 & 1 & 0 & 0 & 0 & 0 & 0 & - \\
\hline & FD & - & 2 & 1 & 0 & 0 & 0 & 0 & 0 & 0 & - \\
\hline Leprosy & Aq. & - & 2 & 0 & 0 & 0 & 0 & 0 & 0 & 0 & - \\
\hline$(\mathrm{n}=2)$ & FD & - & 2 & 0 & 0 & 0 & 0 & 0 & 0 & 0 & - \\
\hline Malaria & Aq. & - & 2 & 2 & 0 & 0 & 0 & 0 & 0 & 0 & - \\
\hline$(n=2)$ & FD & - & 2 & 1 & 0 & 0 & 0 & 0 & 0 & 0 & - \\
\hline $\mathrm{NE}$ & Aq. & - & 4 & 2 & 1 & 0 & 0 & 0 & 0 & 0 & - \\
\hline $\begin{array}{l}\text { Control } \\
(\mathrm{n}=5)\end{array}$ & FD & - & 2 & 1 & 0 & 0 & 0 & 0 & 0 & 0 & - \\
\hline \multicolumn{12}{|c|}{$\begin{array}{l}\text { *Titre: The titre is the highest dilution at which agglutination is visible. Agglutination shows either a blue mat or enlarge blue dot. } \\
\text { The results of the DAT test are given as the reciprocal of the serum dilutions at which agglutination was visible. } \\
\text { Key words: Ag. = antigen; } \mathrm{n}=\text { number; Aq. = aqueous; FD = freeze dried; NE = non endemic. }\end{array}$} \\
\hline \multirow{3}{*}{\multicolumn{2}{|c|}{ ANTIGEN }} & SERA & \multicolumn{9}{|c|}{ TITRE } \\
\hline & & & \multicolumn{5}{|c|}{ Day zero } & \multicolumn{4}{|c|}{ Day 14} \\
\hline & & & \multicolumn{2}{|c|}{$4^{\circ} \mathrm{C}$} & \multicolumn{3}{|c|}{ RT } & \multicolumn{2}{|c|}{$4^{\circ} \mathrm{C}$} & \multicolumn{2}{|l|}{ RT } \\
\hline \multirow{2}{*}{\multicolumn{2}{|c|}{ Aqueous }} & Positive & \multirow{2}{*}{\multicolumn{2}{|c|}{$1: 102400$}} & \multicolumn{3}{|c|}{$1: 102400$} & \multicolumn{2}{|c|}{$1: 102400$} & \multicolumn{2}{|l|}{ NW } \\
\hline & & Negative & & & \multirow{2}{*}{\multicolumn{3}{|c|}{$\begin{array}{l}<1: 100 \\
1: 102400\end{array}$}} & \multicolumn{2}{|c|}{$<1: 100$} & \multicolumn{2}{|l|}{ NW } \\
\hline \multirow{2}{*}{\multicolumn{2}{|c|}{ Freeze Dried ${ }^{* *}$}} & Positive & \multicolumn{2}{|c|}{$1: 102400$} & & & & \multirow{2}{*}{\multicolumn{2}{|c|}{$\begin{array}{l}1: 102400 \\
<1: 100\end{array}$}} & \multirow{2}{*}{\multicolumn{2}{|c|}{$\begin{array}{l}1: 102400 \\
<1: 100\end{array}$}} \\
\hline & & Negative & \multicolumn{2}{|c|}{$<1: 100$} & \multicolumn{3}{|c|}{$\begin{array}{l}1.102400 \\
<1: 100\end{array}$} & & & & \\
\hline
\end{tabular}

Table 3: Evaluation of Freeze Dried and Aqueous antigen in serodiagnosis of KA PKDL and HIV-KA co-infection patients and control by

\begin{tabular}{|c|c|c|c|c|c|c|c|}
\hline \multirow[t]{2}{*}{ Samples types } & & \multirow[t]{2}{*}{ Antigen } & \multicolumn{5}{|c|}{$\begin{array}{l}\text { DILUTIONS } \\
\text { Range of titre expressed by different sera samples }\end{array}$} \\
\hline & & & $<400$ & 400 & 800 & 1600 & $\geq 3200$ \\
\hline \multirow{2}{*}{$\begin{array}{l}\text { Kala-azar: } \\
\text { pre-treatment }\end{array}$} & \multirow[t]{2}{*}{10} & Aq. & 10 & 10 & 10 & 7 & 6 \\
\hline & & FD & 10 & 10 & 8 & 7 & 6 \\
\hline \multirow{2}{*}{$\begin{array}{l}\text { Kala-azar: } \\
\text { post-treatment. }\end{array}$} & \multirow[t]{2}{*}{10} & Aq. & 10 & 10 & 10 & 6 & 2 \\
\hline & & FD & 10 & 10 & 10 & 10 & 9 \\
\hline \multirow[t]{2}{*}{ HIV-KA co-infection } & \multirow[t]{2}{*}{1} & Aq. & 1 & 1 & 1 & 1 & 1 \\
\hline & & FD & 1 & 1 & 1 & 1 & 0 \\
\hline \multirow[t]{2}{*}{ PKDL } & \multirow[t]{2}{*}{5} & Aq. & 5 & 5 & 5 & 3 & 1 \\
\hline & & FD & 5 & 5 & 5 & 5 & 4 \\
\hline
\end{tabular}

The results of the DAT test are given as the reciprocal of the serum dilutions at which agglutination was visible.

Key words: tt. = treatment; Aq. = aqueous; FD = freeze dried

assayed as well as specificity analysis for both antigens from patients suffering from filariasis, leprosy and malaria (Table 1) to give additional support to the above data. A $100 \%$ correlation of results was observed with both the antigen in the dilution range 1:100-1:400 with active KA sera. However, among healthy subjects, $20 \%$ showed positive results at some lower dilutions in freeze dried and aqueous antigens respectively. For FD antigen 1:400 was found optimal because at this dilution, $100 \%$ healthy subjects showed negative results. For aqueous antigen, 1:800 was found optimal because at this dilution all healthy subjects showed negative results. The other important observation, which was noticed, that aqueous antigen always showed 1-2 fold more reading than freeze dried antigen with same sera. Any possible explanation could be made at this point. Once this cut off titre was determined, the sensitivity and specificity was analysed. At 1:400 dilution the aqueous antigen showed $100 \%$ sensitivity and $81.82 \%$ specificity were as freeze dried antigen demonstrated $100 \%$ sensitivity and specificity. The results once again confirmed the earlier finding that sera dilution 1:400 and 1:800 of FD and aqueous antigen respectively should be considered as a DAT diagnostic titre to differentiate not only active Kala-azar from healthy subject of non-endemic zone but to Kalaazar from other cross reactive controls (.....), because at this titre neither FD nor aqueous antigen showed any positive reaction in any sera. Harith et al. ${ }^{[2]}$ suggested 1:3200 for aqueous antigen where as Meridith et al. ${ }^{[5]}$ demonstrated 1:1600 for FD antigen by DAT in diagnosis of active VL cases to control. 
The storage of antigen is another critical issue. It was observed that the fresh aqueous antigen if kept at room temperature lost almost all the antigenic activity. This loss of activity was not drastic with the same antigen when it was kept at $4^{\circ} \mathrm{C}$. In contrary the freezedried antigen was found highly stable, do not show any change in activity at $4^{\circ} \mathrm{C}$ for 14 days and at RT, 1-fold reduction in activity. These results clearly demonstrate that for any storage, fieldwork and where cold chain cannot be carried, freeze-dried antigen should be used for diagnosis of Kala-azar cases.

Using the two antigens, DAT antibody titres in 10 cases each representing active VL cases and VL cases after completion of SAG therapy and 5 PKDL subjects were evaluated. In active KA sera antibody positivity at dilution of $1: 800$ in 3 and $1: 1600$ in 1 and $\geq 1: 3200$ in 6 cases were recorded with aqueous antigen. Antibody titre in same seraby FD antigen 1:400 dilution in 2, $1: 800$ in $2,1: 1600$ in 2 and $\geq 3200$ dilution in 5 sera samples of this category Among the 10 Kala-azar samples, reading obtained using using FD antigen were identical in $2(20 \%)$ lower in $8(10 \%)$ and higher in none $(0 \%)$ compared to the aqueous antigen.. The performance of both the aqueous and FD antigen was more or less similar except that the end point titre was 2/3 dilutions lower than the aqueous antigen. At 1: 800 cut-off of aqueous antigen when considered, 8/10 of these KA sera samples gave positive reaction with both the antigens with no cross reactivities with other diseased samples. This indicated that even at cut-off of aqueous antigen the sensitivity and specificity of FD antigen was $80 \%$ and $100 \%$ respectively, thereby compared well with aqueous antigen. Pre and post treatment antibody kinetics with aqueous and FD antigens as studied however reflected sharp differences in discriminating efficacy of these antigens. Evaluation made on post treated sera of same patients showed that decline in antibody titre with aqueous antigen was more rapid as compared to FD antigen. While with aqueous antigen, antibody titre was 1:800 in 4 post-treated sera, 6 sera in this category were at or above cut off for aqueous antigen (1:800). As compared to aqueous, the FD antigen did not show much decline in titre pattern. As a result, all the 10 post treated sera showed antibody titre at or above the cut off set for FD antigen (1:400). Readings obtained using FD antigens in this category were identical in $2(20 \%)$ while it recorded a 2-3 dilutions higher titre in remaining sera when compared to aqueous antigen. All of the 8 post treated sera samples of KA patients showing a high titre with FD antigen were tested at the completion of the SAG therapy. Identical readings obtained using FD antigen in sera samples from two patients who had completed fungizone treatment.

Difference in the performance of these antigens on KA and PKDL sera was clearly shown. Antibody titre was low (1:800-1:3200) with aqueous form in PKDL sera but FD antigen remarkably gave a better result when the same sera were observed positive at higher dilutions. Two of five sera samples of PKDL patients positive at 1:1600 dilution with aqueous antigen were found positive one each at 1:12800 and 1:25600 dilutions respectively with FD antigen. Therefore unlike KA the end point titre was 2-3 dilutions higher in sera samples of PKDL patients. As earlier reported, in HIV-Leishmania co-infection case the serological results are variable ranging from $6-82 \%$ positivity ${ }^{[7,8]}$. In one HIV-VL co-infection cases tested we observed high DAT positive titre with both the antigen. Earlier Gradoni et $a l .{ }^{[9]}$ ) reported that HIV patients aquiring their Leishmania infection before being infected with HIV usually produce high antibody titre. Taken this into consideration we suggest doing more studies of similar kind in future to get mote insight on HIV prevalence in Leishmania endmic areas in the Indian state of Bihar where such cases are high on rise.

From the preliminary results of evaluation of DAT using FD antigen we conclude that through in preparation of FD antigen one additional step is required than the aqueous antigen but FD antigen is more stable, equally sensitive and very specific for the diagnosis of VL, PKDL and even HIV-VL co-infection (data not given). So the FD antigen should be used for routine mass diagnosis of Leishmania cases in labs field conditions, because this antigen is very stable at RT after 14 days without any reduction in antigenic activity.

\section{ACKNOWLEDGEMENT}

Technical Assistance rendered by Mr. Arvind Prasad Technical Assistant, Immunology Department, RMRIMS (ICMR) is gratefully acknowledged.

\section{REFERENCES}

1. Adhya, S., M. Chatterjee, M.Q. Hassan, S. Mukherjee and S. Sen, 1995. Detection of Leishmania in the blood of early Kala-azar patients with the aid of the polymerase chain reaction. Trans. R. Soc. Trop. Med. Hyg., 89: 622-24.

2. Harith, A.E., A.H.J. Kolk, J. Leeuwenburg, R. Murgai, E. Huigen, J. Jelsma and P.A. Kagar, 1988. Improovement of direct agglutination test for field studies of visceral leishmaniasis. J. Clin. Microbiol., 26: 1321-1325.

3. Singla, N., G.S. Singh, S. Sundar and V.K. Vinayak, 1993. Evaluation of the direct agglutination test as an immunodiagnostic tool for kala-azar in India. Trans. R. Soc. Trop. Med. Hyg., 87: 276-8. 
4. Sharma, M.C., S. Bimal, A. Ranjan, V.N.R. Das, A.K. Gupta, N. Kumar and S.K. Kar, 2000. Relevance of direct agglutination test in seroepidemiological survey of Kala-azar in epidemic foci of Bihar. J. Commun. Dis., 32: 117122.

5. Meredith, S.E.O., N.C.M. Kroon, E. Sondorp, J. Seaman, M.G.A. Goris, C.W. Van Ingen, H. Oosting, G.J. Schoone, W.L. Terpstra and L. Oskam, 1995. Leish-KIT, a stable direct agglutination test based on freeze-dried antigen for serodiagnosis of visceral leishmaniasis. J. Clin. Microbiol., 33:1742-1745.

6. Harith, A.E., R.J. Slappendel, I. Reiter, F.V. Knaper, P. Korte, E. Huigen and A.H.J. Kolk, 1989. Application of a direct agglutination test for detection of specific anti-Leishmania antibodies in a canine reservoir. J. Clin. Microbiol., 27: 2252.
7. Gari-Toussaint, M., A. Lelievre, P. Marty and Y. Le Fichoux, 1994. Contribution of serological tests to the diagnosis of visceral leishmaniasis in patients infected with the human immunodeficiency virus. Trans. R. Soc. Trop. Med. Hyg., 88: 301-302.

8. Alvar, J., C. Canavate, B. Gutierrez-Solar, M. Jimenez, F. Laguna, R. Lopez-velez, R. Molina and J. Moreno, 1997. Leishmania and human immunodeficiency virus co-infection: the first 10 years. Clin. Microbiol. Rev., 10: 298-319.

9. Gradoni, L., A. Scalone and M. Gramiccia, 1993. HIV-Leishmania co-infection in Italy: serological data as an indication of the sequence of acquisition of the two infections. Trans. R. Soc. Trop. Med. Hyg., 87: 94-96. 